\title{
Measured Probabilities of Detection for 1- and 2 Bitstreams of 2-port Car-roof Antenna in RIMP and Random-LOS
}

\author{
Madeleine Schilliger Kildal ${ }^{1,2}$, Andrés Alayón Glazunov², Jan Carlsson ${ }^{2,3}$, \\ John Kvarnstrand ${ }^{1}$, Amir Majidzadeh ${ }^{4}$ and Per-Simon Kildal ${ }^{3}$ \\ ${ }^{1}$ Bluetest AB, Gothenburg, Sweden \\ ${ }^{2}$ Department of Signals and Systems, Chalmers University of Technology, Gothenburg, Sweden \\ ${ }^{3}$ Electronics department, SP Technical Research Institute of Sweden, Borås, Sweden \\ ${ }^{4}$ Volvo Car Corporation, Gothenburg, Sweden
}

\begin{abstract}
Autonomous cars will in a near future drive around in cities and on highways. Antennas will then be needed to secure the wireless connection to these cars. To be able to test the antennas we have defined two edge environments: the Random Line-of-Sight (LOS) and the Rich Isotropic Multipath (RIMP). This paper shows a throughput performance comparison between measurements and simulations of a car-roof (shark-fin) antenna mounted on a ground plane in both of these environments. The comparison is done for both one and two bitstreams in a $2 \times 2$ MIMO system. The analysis is based on probability of detection (PoD) curves representing the throughput performance with digital threshold receivers.
\end{abstract}

Index Terms-RIMP, reverberation chamber, Random-LOS, anechoic chamber, automotive OTA measurements, threshold receiver.

\section{INTRODUCTION}

The Rich Isotropic Multipath (RIMP) environment has for many years been a known reference environment for characterizing the Over-the-Air (OTA) performance of mobile phones and small antennas [1],[2]. The RIMP environment corresponds to an environment with many scatterers, i.e., rich multipath. However, not all mobile antennas are used in such environments. Moreover, in the future, in order to meet communication requirements, many more antennas than today will be used. This is particularly relevant for future autonomous cars. In addition to the RIMP environment, cars will often drive in Line-of-Sight (LOS) environments, such as on highways. In traditional LOS the angle of arrival (AoA) is fixed. However, cars can be oriented in any direction in the horizontal plane, which will give rise to a $2 \mathrm{D}$ randomness of the AoA. Therefore we call this environment Random-LOS. In [3] a hypothesis was formulated, stating that if an antenna works well in both Random-LOS and RIMP, it will also work well in a real-life environment.

The main concepts for active measurements on a car in both RIMP or Random-LOS environments were presented in [4]. The first initial Random-LOS measurements performed on a car were presented in [5]. Simulations showing how measurement accuracy can be improved by means of a linear array are provided in [6].

The Random-LOS measurement technique resembles at this stage to some degree the two-stage OTA test method [7]. Presently, we measure the radiation pattern to get theoretical comparisons for our active measurements, but in the future this will not be needed. Then, we will be able to perform active measurements directly without the need of cable connections to the modem. There exist also other techniques to perform car-to-infrastructure OTA tests. Ilmenau University of Technology has a test facility where they are looking at emulating realistic radio scenarios for cars [8]. The authors of [9] investigate the measurement uncertainty of a multi-probe setup for characterizing a MIMO antenna on a car.

Following the real-life hypothesis [3], we present here the OTA characterization of a shark-fin antenna in both the RIMP and the Random-LOS environments. The antenna is mounted on a ground plane to emulate the near environment of the antenna when mounted on a car.

\section{METHOD}

Measurements were performed both in an RC (Reverberation Chamber) and an AC (Anechoic Chamber), emulating the RIMP and the Random-LOS environments, respectively. The antenna under test (AUT) was a radome covered shark-fin antenna (provided by Volvo) placed on a square ground plane as shown in Fig. 2. The shark-fin antenna is an array antenna comprising two different antenna elements, a larger one and a smaller one that we denote port 1 and 2, respectively; see Fig. 2. The shark-fin antenna needs to be fed with $12 \mathrm{~V}$ to activate a switch for the smaller element. The power aggregate was placed outside both chambers during the measurements.

To be able to perform active OTA measurements, the sharkfin antenna was connected through cables to a Huawei LTE USB modem dongle (E398). The dongle was placed inside a shielded box (Aeroflex RF Shield, Model 4931) to make sure that the internal antennas of the modem were not used. To control and provide the dongle with electricity it was 


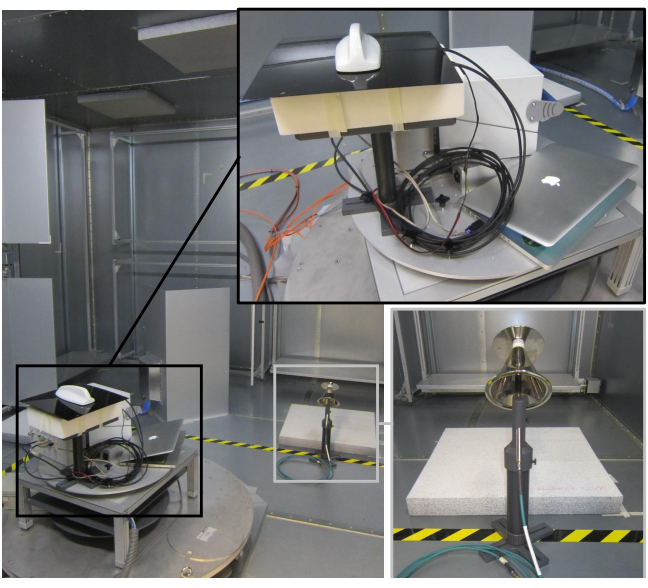

Fig. 1. The measurement setup in the Bluetest RTS90 RC for the RIMP measurements. The shark-fin antenna with ground plane is placed on the turntable together with the shielded box and the laptop. The reference disccone antenna is shown in the bottom right corner of the figure.

connected to a laptop that was placed outside the shielded box.

Throughput measurements were done by using a CMW500 communication tester from Rohde \& Schwarz, which was controlled by software provided by Bluetest. The measurements were performed at LTE band 7 and channel 3100, with the downlink frequency $2.655 \mathrm{GHz}$. The system bandwidth was set to $B_{s}=5 \mathrm{MHz}$, and a downlink and uplink modulation of 16-QAM and QPSK were used respectively. The number of subframes per stream was set to 400 and 25 resource blocks were used. The maximum theoretical and measured throughput was $5.738 \mathrm{Mbps}$ (one bitstream) for both single-input singleoutput (SISO) and single-input multiple-output (SIMO). For the multiple-input multiple-output (MIMO) measurements, with two bitstreams, the maximum throughput was the double of the SISO and SIMO measurements, i.e., $11.476 \mathrm{Mbps}$. The MIMO measurements were done using transmission mode 3 (TM 3). All the measurements were done with the following modulation and coding scheme (MCS) indices; subframe 0 used MCS 13, subframe 1-4 and 6-9 used MCS 14 and subframe 5 was not used.

\section{A. RIMP measurements}

The Bluetest RTS90 RC (dimensions $3.3 \mathrm{~m} \times 2.55 \mathrm{~m} \times$ $4.2 \mathrm{~m}$ ) was used for the RIMP measurements. The shark-fin antenna with ground plane was placed on a holder on the turntable, see Fig. 1. The shielded box with the dongle inside was also placed on the turntable together with the laptop.

Reference measurements accounting for chamber losses were performed with the disc-cone antenna shown in Fig. 1 and a vector network analyzer (Rohde \& Schwarz ZNB $8,9 \mathrm{kHz}-8.5 \mathrm{GHz})$. The disc-cone antenna efficiency was known. A frequency stirring of $5 \mathrm{MHz}$ was used for the reference measurement, which is equal to the system bandwidth. The losses of the cables between the shark-fin antenna and the dongle were manually compensated for after the measurements

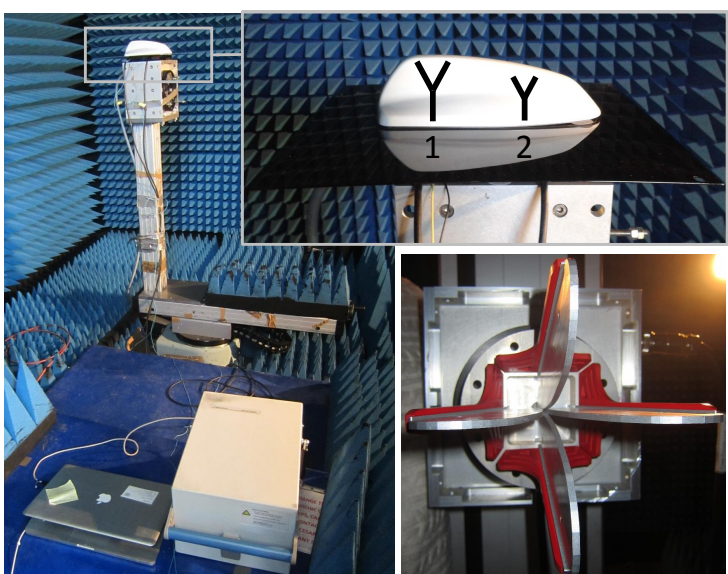

Fig. 2. The measurement setup in the AC at Chalmers for the RandomLOS measurements. The shark-fin antenna with ground plane is placed on the turntable; and the shielded box together with the laptop is placed on the floor close to the door. The numbers on the shark-fin denotes the two antenn elements. The larger one is denoted 1 and the smaller one 2. The quadridge horn antenna used as the chamber antenna is shown at the bottom right corner of the figure.

had been done.

The chamber was loaded with eight evenly distributed absorbers (dimensions $60 \mathrm{~cm} \times 60 \mathrm{~cm} \times 6 \mathrm{~cm}$ ) to get a delay spread of $94 \mathrm{~ns}$. This corresponds to a coherence bandwidth of $B_{c}=6 \mathrm{MHz}$ [10]. The throughput was measured during continuous mode stirring in the reverberation chamber and with power steps of $1 \mathrm{dBm}$. Every throughput value in the throughput curve is computed by averaging over 120 throughput samples.

The theoretical RIMP data was created according to [11] by using

$$
\operatorname{PoD}\left(P_{\mathrm{av}} / P_{\mathrm{th}}\right)=1-\operatorname{CDF}\left(P_{\mathrm{th}} / P_{\mathrm{av}}\right),
$$

where $\mathrm{PoD}$ is the probability of detection, $P_{\mathrm{av}}$ is the averaged received power, $P_{\mathrm{th}}$ is the threshold level and CDF is the cumulative distribution function. The threshold level was obtained by performing a conducted measurement, where the modem in the shielded box was directly connected to the communication tester by cables. The system bandwidth and coherence bandwidth of the chamber are almost the same. This will not give rise to any frequency diversity channels according to the model in [11], since $N_{\mathrm{fd}}=\operatorname{NINT}\left(B_{s} / B_{c}\right)$, where NINT is the nearest integer function. Maximal ratio combining (MRC) of two uncorrelated antenna elements was used to get the SIMO curve. The theoretical MIMO curve for detecting two bitstreams was obtained assuming Zero Forcing (ZF) according to [12]. The detection of two bitstreams corresponds to the detection of the weakest stream [13], which is used in the $\mathrm{ZF}$ algorithm. The total radiation efficiencies of the antenna elements were measured in the $\mathrm{RC}$ and used for correcting the theoretical RIMP curves. 


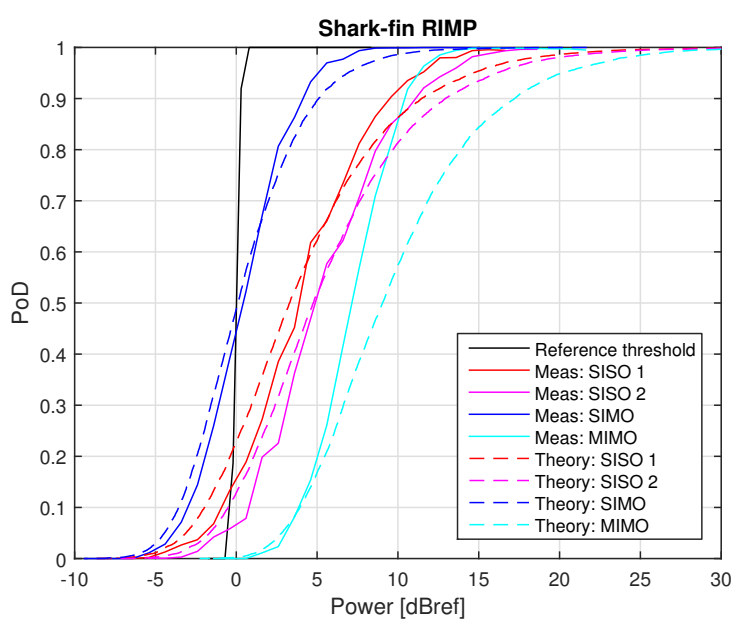

(a)

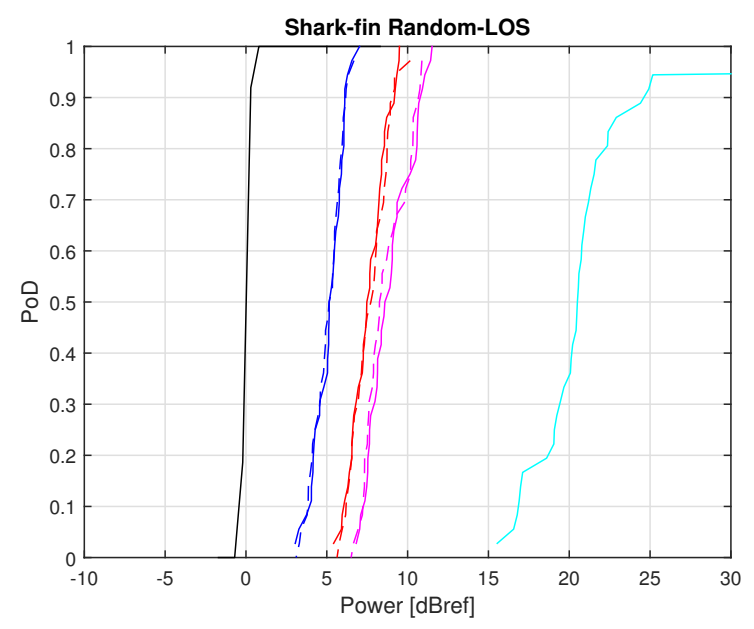

(b)

Fig. 3. Measured and theoretical PoD curves for the shark-fin antenna in RIMP and Random-LOS. The results are normalized according to a reference threshold. The SISO and SIMO curves corresponds to one bitstream and the MIMO curves correspond to two bitstreams. (a) The relative throughput/PoD level from the RIMP measurements. The MIMO curve has in reality higher throughput compared to the SISO and SIMO curve, but this is not shown since the curves are normalized to their maximum throughput. The reference threshold corresponds to the conducted reference threshold of the dongle.(b) The PoD from the Random-LOS measurements. The reference threshold corresponds to the threshold of the reference bowtie antenna, with a realized gain of $3 \mathrm{~dB}$ [5], facing the chamber antenna.

\section{B. Random-LOS measurements}

The Random-LOS environment was realized in an anechoic chamber (dimensions $2.0 \mathrm{~m} \times 3.5 \mathrm{~m} \times 5.5 \mathrm{~m}$ ) at Chalmers University of Technology. The shark-fin antenna with ground plane was placed on top of the turntable, see Fig. 2. A quadridge horn antenna (ETS Lindgren Open Boundary Quadridge Horn, Model 3164-05) was used as a chamber antenna emulating a base station antenna. The two antennas were placed at the same height, with a distance of $3.8 \mathrm{~m}$ between them.

A reference measurement was first performed with the bowtie antenna on the turntable. Both the bowtie antenna and the chamber antenna were placed at the same height, with a distance of $3.7 \mathrm{~m}$ between them. The transmission, $S_{21}$, was measured with a vector network analyzer (Agilent E8363B PNA), and used as reference. The measurement setups for the reference (bowtie antenna) and the shark-fin measurement were the same except for the antennas and their positions on the turntable.

The throughput was measured at discrete angles every $10^{\circ}$ in the horizontal plane over a whole $360^{\circ}$ turn of the turntable. At each angle the power was swept, in steps of $0.5 \mathrm{dBm}$, over a range of values covering throughput levels from $0 \%$ to $100 \%$.

The theoretical throughput curves corresponding to the Random-LOS case were obtained by measuring the radiation patterns of the 2-port shark-fin in the horizontal plane. The radiation pattern measurements were performed in the same $\mathrm{AC}$, with the same measurement setup (except for the shielded box, dongle and the laptop), as in the active measurements. The measurements were performed with the same vector network analyzer as for the bowtie reference. The radiation patterns were used to compute the CDFs of the received power and the corresponding PoDs according to (1). The SIMO curve was obtained by applying the MRC algorithm to the radiation patterns of the two antenna elements of the shark-fin antenna. Given that both antenna elements are vertically polarized, no theoretical 2-bitstream MIMO throughput can be computed in the Random-LOS case [14].

\section{RESUlts}

The relative throughput curves, or equivalently the PoD curves, from the RIMP measurements are shown in Fig. 3a. Each throughput curve is an average over all the stirrer positions in the RC. Indeed, at every static position of the stirrers a steep throughput curve is measured at the threshold level [15]. It is also worth noting that with MIMO we transmit two bitstreams and therefore the maximum absolute throughput is higher for MIMO. However, since we present PoD curves the maximum value of both SISO, SIMO and MIMO is the same. The RIMP throughput curves have been normalized to the conducted measurement threshold of the modem dongle $(-93.3 \mathrm{dBm})$, which corresponds to the $0 \mathrm{dBref}(\mathrm{dB}$ relative a reference) power level in Fig. 3a.

The theoretical RIMP curves (obtained according to [11] and [12]) are shown as dotted lines. In order to obtain the theoretical curves, the total radiation efficiencies for the sharkfin antenna ports have been taken into account. The total radiation efficiencies are $-1.7 \mathrm{~dB}$ and $-3.1 \mathrm{~dB}$ for antenna ports 1 and 2, respectively. We can see that all the measured RIMP curves are steeper than the theoretical ones. This means that we have a diversity gain in the measured case that we have not taken into account for in our theoretical model. The difference is in particularly large for the MIMO curve. This difference seams to be due to a diversity gain that we 
can not explain yet, but we are working on some different explanations, such like cyclic delay diversity.

In the Random-LOS environment the throughput curve for every measured angle will take the form of a threshold curve [15]. The threshold level corresponding to each rotation angle is obtained as the power corresponding to the $50 \%$ level of detection threshold [5]. The CDF is then formed from the estimated threshold levels. This CDF corresponds to the PoD of the level of detection thresholds. The PoD curves are normalized to a reference threshold corresponding to the threshold of the reference bowtie antenna used for the reference measurements. This means that we plot the curves relative to the performance of a known antenna with a realized gain of $3 \mathrm{~dB}$. This value is low because of the $180^{\circ}$ hybrids used to feed the bowtie differentially, as explained in [5]. The final PoD curves are presented in Fig. $3 b$.

The PoD curves in Fig. $3 b$ are steeper than the PoD curves presented in [5], where a whole car is used. This difference is caused by the influence of the shape of the car on the radiation pattern of the shark-fin antenna. The shape of the car causes larger azimuthal variations, and therefore a less steep PoD curve.

The theoretical Random-LOS curves, obtained from the radiation pattern, are shown as dotted lines in Fig. $3 \mathrm{~b}$. A comparison between the median value of the radiation pattern of shark-fin port 1 and the bowtie reference showed that the shark-fin performs $7.7 \mathrm{~dB}$ worse. All the theoretical PoDs from the radiation pattern were therefor shifted to correspond to this difference. The $7.7 \mathrm{~dB}$ value corresponds to the difference in realized gain between the directive bowtie antenna and the median value of the omnidirectional shark-fin antenna. The theoretical Random-LOS curves are almost overlapping with the measured PoD curves. The theoretical 2-bitstream MIMO curve does not exist, since in theory we cannot get MIMO performance in Random-LOS without dual polarization on the receiver. Also, it is clearly seen in the RandomLOS measurement curves that there is needed at least $13 \mathrm{~dB}$ more power, at the $90 \%$ PoD level, to be able to get two bitstreams in a Random-LOS environment compared to the worst one bitstream case. These $13 \mathrm{~dB}$ are basically a measure of how non-orthogonal the two antenna ports are. If they were orthogonal the ratio would be $0 \mathrm{~dB}$. To be able to achieve two bitstreams in Random-LOS we would need to have an antenna with two orthogonally polarized ports on the receiving side. This is hard to get with an antenna on the roof of a car, since the roof will act as a large ground plane and prevent a realization of horizontal polarization.

The diversity gain at the $90 \% \mathrm{PoD}$ level is larger in RIMP than in Random-LOS. In Random-LOS we get an array gain/power gain of $3 \mathrm{~dB}$ because we receive the same signal at the two antenna ports (correlated signals). However, in RIMP we get a diversity gain of $5.5 \mathrm{~dB}$, which is due to both the power gain of having two antennas and the uncorrelated signals that we receive on the two ports.

\section{CONCLUSION}

The RIMP and the Random-LOS environments are two very different environments. For a car antenna to perform well in real life we want it to perform well in both these environments. This paper shows that it is very hard to get 2bitstream MIMO performance in a Random-LOS environment when the antenna is mounted on a ground plane. Polarization diversity on the receiving antenna is needed in order to get two bitstreams. However, it is hard to get horizontal polarization of an antenna on a car-roof, since the roof of the car acts as a large ground plane. In the RIMP environment, on the other hand, we can easily get 2-bitstream MIMO performance with the two antenna ports. The measured results for one bitstream show good agreement with simulations, whereas we need to work a bit more on the model for the two bitstream case.

\section{ACKNOWLEDGMENT}

We would like to thank Mats Kristoffersen and Anton Skårbratt at Bluetest who helped with questions and software issues. The project is partly supported by the Swedish Research Council VR, through an industrial $\mathrm{PhD}$ project.

\section{REFERENCES}

[1] P.-S. Kildal and K. Rosengren, "Correlation and capacity of MIMO systems and mutual coupling, radiation efficiency, and diversity gain of their antennas: simulations and measurements in a reverberation chamber," IEEE Communications Magazine, vol. 42, no. 12, pp. 104112, Dec. 2004.

[2] P.-S. Kildal, C. Orlenius, and J. Carlsson, "OTA Testing in Multipath of Antennas and Wireless Devices With MIMO and OFDM," Proceedings of the IEEE, vol. 100, no. 7, pp. 2145-2157, Jul. 2012.

[3] P.-S. Kildal and J. Carlsson, "New approach to OTA testing: RIMP and pure-LOS reference environments amp; a hypothesis," in 2013 7th European Conference on Antennas and Propagation (EuCAP), Apr. 2013, pp. 315-318.

[4] P.-S. Kildal, A. A. Glazunov, J. Carlsson, and A. Majidzadeh, "Costeffective measurement setups for testing wireless communication to vehicles in reverberation chambers and anechoic chambers," in 2014 Conference on Antenna Measurements Applications (CAMA), Nov. 2014, pp. 1-4.

[5] M. S. Kildal, J. Kvarnstrand, J. Carlsson, A. A. Glazunov, A. Majidzadeh, and P.-S. Kildal, "Initial Measured OTA Throughput of 4g LTE Communication to Cars with Roof-Mounted Antennas in 2d RandomLOS," in 2015 International Symposium on Antennas and Propagation (ISAP), 2015.

[6] A. A. Glazunov, P.-S. Kildal, and M. S. Kildal, "Devising a Horizontal Chamber Array for Automotive OTA Tests in Random Line-Of-Sight," in 2015 International Symposium on Antennas and Propagation (ISAP), 2015.

[7] Y. Jing, X. Zhao, H. Kong, S. Duffy, and M. Rumney, "Two-Stage Over-the-Air (OTA) Test Method for LTE MIMO Device Performance Evaluation," International Journal of Antennas and Propagation, vol. 2012, p. e572419, Jul. 2012.

[8] R. Sharma, C. Schneider, W. Kotterman, G. Sommerkorn, P. Grosse, F. Wollenschlager, G. Del Galdo, M. Hein, and R. Thoma, "Over-theair testing of Car-to-Car and car-to-infrastructure communication in a virtual electromagnetic environment," in IECON 2013 - 39th Annual Conference of the IEEE Industrial Electronics Society, Nov. 2013, pp. 6897-6902.

[9] M. Nilsson, P. Hallbjorner, N. Araback, B. Bergqvist, T. Abbas, and F. Tufvesson, "Measurement Uncertainty, Channel Simulation, and Disturbance Characterization of an Over-the-Air Multi-Probe Setup for Cars at $5.9 \mathrm{GHz}$," IEEE Transactions on Industrial Electronics, vol. PP, no. 99 , pp. 1-1, 2015. 
[10] X. Chen, P.-S. Kildal, C. Orlenius, and J. Carlsson, "Channel Sounding of Loaded Reverberation Chamber for Over-the-Air Testing of Wireless Devices: Coherence Bandwidth Versus Average Mode Bandwidth and Delay Spread,' IEEE Antennas and Wireless Propagation Letters, vol. 8, pp. 678-681, 2009, corrections in IEEE Antennas and Wireless Propagation Letters, vol. 12, 2013

[11] A. Hussain, P.-S. Kildal, and A. A. Glazunov, "Interpreting the Total Isotropic Sensitivity and Diversity Gain of LTE-Enabled Wireless Devices From Over-the-Air Throughput Measurements in Reverberation Chambers," IEEE Access, vol. 3, pp. 131-145, 2015.

[12] X. Chen, P.-S. Kildal, and M. Gustafsson, "Characterization of Implemented Algorithm for MIMO Spatial Multiplexing in Reverberation Chamber,' IEEE Transactions on Antennas and Propagation, vol. 61 , no. 8, pp. 4400-4404, Aug. 2013.

[13] P. Kildal, X. Chen, M. Gustafsson, and Z. Shen, "MIMO Characterization on System Level of $5 \mathrm{~g}$ Microbase Stations Subject to Randomness in LOS," IEEE Access, vol. 2, pp. 1062-1075, 2014.

[14] A. Razavi, A. A. Glazunov, P.-S. Kildal, and J. Yang, "Investigation of Polarization Deficiencies in SIMO Systems in Random-LOS Propagation Channels," in 2015 International Symposium on Antennas and Propagation (ISAP), 2015.

[15] P.-S. Kildal, A. Hussain, X. Chen, C. Orlenius, A. Skarbratt, J. Asberg, T. Svensson, and T. Eriksson, "Threshold Receiver Model for Throughput of Wireless Devices With MIMO and Frequency Diversity Measured in Reverberation Chamber," IEEE Antennas and Wireless Propagation Letters, vol. 10, pp. 1201-1204, 2011. 